\title{
A NEW REGULARITY CRITERION FOR THE NAVIER-STOKES EQUATIONS IN TERMS OF THE GRADIENT OF ONE VELOCITY COMPONENT *
}

\author{
YONG $\mathrm{ZHOU}^{\dagger}$
}

\begin{abstract}
In this paper we consider the regularity criteria for the weak solutions to the NavierStokes equations in $\mathbb{R}^{3}$. It is proved that if the gradient of any one component of the velocity field belongs to $L^{\alpha, \gamma}$ with $2 / \alpha+3 / \gamma=3 / 2,3 \leq \gamma<\infty$, then the weak solution actually is strong.
\end{abstract}

1. Introduction. We consider the following Cauchy problem for the incompressible Navier-Stokes equations in $\mathbb{R}^{3} \times(0, T)$

$$
\left\{\begin{array}{l}
\frac{\partial u}{\partial t}+u \cdot \nabla u+\nabla p=\Delta u \\
\operatorname{div} u=0 \\
u(x, 0)=u_{0}(x)
\end{array}\right.
$$

where $u=\left(u_{1}(x, t), u_{2}(x, t), u_{3}(x, t)\right)$ is the velocity field, $p(x, t)$ is a scalar pressure, and $u_{0}(x)$ with $\operatorname{div} u_{0}=0$ in the sense of distribution is the initial velocity field.

The study of the incompressible Navier-Stokes equations in three space dimensions has a long history. In the pioneering work [10] and [7], Leray and Hopf proved the existence of its weak solutions $u(x, t) \in L^{\infty}\left(0, T ; L^{2}\left(\mathbb{R}^{3}\right)\right) \cap L^{2}\left(0, T ; H^{1}\left(\mathbb{R}^{3}\right)\right)$ for given $u_{0}(x) \in L^{2}\left(\mathbb{R}^{3}\right)$. But the uniqueness and regularity of the Leray-Hopf weak solutions are still big open problems. In [12], Scheffer began to study the partial regularity theory of the Navier-Stokes equations. Deeper results were obtained by Caffarelli, Kohn and Nirenberg in [2]. Further result can be found in [17] and references there in.

On the other hand, the regularity of a given weak solution $u$ can be shown under additional conditions. In 1962, Serrin [13] proved that if $\mathrm{u}$ is a Leray-Hopf weak solution belonging to $L^{\alpha, \gamma} \equiv L^{\alpha}\left(0, T ; L^{\gamma}\left(\mathbb{R}^{3}\right)\right)$ with $2 / \alpha+3 / \gamma \leq 1,2<\alpha<\infty, 3<$ $\gamma<\infty$, then the solution $u(x, t) \in C^{\infty}\left(\mathbb{R}^{3} \times(0, T)\right)$. From then on, there are many criterion results added on $u$. In [18] and [5], von Wahl and Giga showed that if $u$ is a weak solution in $C\left([0, T) ; L^{3}\left(\mathbb{R}^{3}\right)\right)$, then $u(x, t) \in C^{\infty}\left(\mathbb{R}^{3} \times(0, T)\right)$; Struwe [16] proved the same regularity of $u$ in $L^{\infty}\left(0, T ; L^{3}\left(\mathbb{R}^{3}\right)\right.$ provided $\sup _{0<t<T}\|u(x, t)\|_{L^{3}}$ is sufficiently small and Kozono and Sohr [8] obtained the regularity for the weak solution $u(x, t) \in C^{\infty}\left(\mathbb{R}^{3} \times(0, T)\right)$ provided $u(x, t)$ is left continuous with respect to $L^{3}$-norm for every $t \in(0, T)$. Recently Kozono and Taniuchi [9] showed that if a Leray-Hopf weak solution $u(x, t) \in L^{2}(0, T ; B M O)$, then $u(x, t)$ is actually a strong solution of $(1)$ on $(0, T) . L^{\alpha, \gamma}$ is defined by

$$
\|u\|_{L^{\alpha, \gamma}}= \begin{cases}\left(\int_{0}^{t}\|u(., \tau)\|_{L^{\gamma}}^{\alpha} d \tau\right)^{1 / \alpha} & \text { if } 1 \leq \alpha<\infty \\ \operatorname{ess} \sup _{0<\tau<t}\|u(., \tau)\|_{L^{\gamma}} & \text { if } \alpha=\infty\end{cases}
$$

\footnotetext{
${ }^{*}$ Received November 21, 2002; accepted for publication January 14, 2003. This work is partically supported by Hong Kong RGC Earmarked Grants CUHK-4219-99P and CUHK-4279-00P.

${ }^{\dagger}$ The Institute of Mathematical Sciences and Department of Mathematics, The Chinese University of Hong Kong, Shatin, N.T., Hong Kong (yzhou@math.cuhk.edu.hk).
} 
where

$$
\|u(., \tau)\|_{L^{\gamma}}= \begin{cases}\left(\int_{\mathbb{R}^{3}}|u(x, \tau)|^{\gamma} d x\right)^{1 / \gamma} & \text { if } 1 \leq \gamma<\infty \\ \operatorname{ess} \sup _{x \in \mathbb{R}^{3}}|u(x, \tau)| & \text { if } \gamma=\infty\end{cases}
$$

The point is that $\left\|u_{\lambda}\right\|_{L^{\alpha, \gamma}}=\|u\|_{L^{\alpha, \gamma}}$ holds for all $\lambda>0$ if and only if $2 / \alpha+3 / \gamma=1$, where $u_{\lambda}(x, t)=\lambda u\left(\lambda x, \lambda^{2} t\right), p_{\lambda}(x, t)=\lambda^{2} p\left(\lambda x, \lambda^{2} t\right)$ and if $(u, p)$ solves the NavierStokes equations, then so does $\left(u_{\lambda}, p_{\lambda}\right)$ for all $\lambda>0$. Usually we say that the norm $\|u\|_{L^{\alpha, \gamma}}$ has the scaling dimension zero for $2 / \alpha+3 / \gamma=1[2]$.

Sohr [14] extended Serrin's regularity criterion by introducing Lorentz space in both time and spatial direction, $u \in L^{s, r}\left(0, T ; L^{q, \infty}\right)$ with $2 / s+3 / q=1,3<q<\infty$, $2<s \leq r<\infty$, here $L^{p, q}$ is Lorentz space, for weak solutions which satisfy the strong energy inequality. Later on, Sohr [15] extended Serrin's regularity class for weak solutions of the Navier-Stokes equations replacing the $L^{q}$-space by Sobolev spaces of negative order, $u \in L^{s}\left(0, T ; H^{-\alpha, q}\right)$ with $2 / s+3 / q=1-\alpha, 3<q<\infty, 2<s<\infty$, for $0 \leq \alpha<1$.

Zhou [21] proved the regularity of the Leray-Hopf weak solution by adding the Serrin's regularity criterion only on two components of the velocity field. Also in [21], the author gave a regularity criterion by adding condition on one velocity component, say, $u_{3} \in L^{\alpha, \gamma}$ with $2 / \alpha+3 / \gamma \leq 1 / 2$ for $\gamma>6$.

One can find that if $2 / \alpha+3 / \gamma=2$, both $\|\nabla u\|_{L^{\alpha, \gamma}}$ and $\|p\|_{L^{\alpha, \gamma}}$ have scaling dimension zero. Related to this point, Beirão da Veiga [1] proposed the regularity criterion on $\nabla u$, which states that if a weak solution $u(x, t)$ satisfies $\nabla u \in L^{\alpha, \gamma}, 2 / \alpha+$ $3 / \gamma \leq 2,3 / 2<\gamma<\infty$, then $u(x, t) \in C^{\infty}\left(\mathbb{R}^{3} \times(0, T)\right)$. Chae and Choe [3] improved Beirão da Veiga's condition by imposing that only on the two components of the vorticity field. Very recently, Zhou [21] proved that if a Leray-Hopf weak solution satisfies $\nabla u_{3} \in L^{\alpha, \gamma}$ with $2 / \alpha+3 / \gamma \leq 3 / 2,2<\gamma<3$, or $\left\|\nabla u_{3}\right\|_{L^{\infty, 2},}$ is sufficiently small, then the weak solution is strong.

In this paper, we want to prove the analogous result for $\gamma \geq 3$. More precisely, our main theorem reads

THEOREM 1. Suppose $u_{0} \in H^{1}\left(\mathbb{R}^{3}\right)$, and div $u_{0}=0$ in the sense of distribution. Assume that $u(x, t)$ is a Leray-Hopf weak solution of (1) in $(0, T)$. If $\nabla u_{3} \in L^{\alpha, \gamma}$ with $2 / \alpha+3 / \gamma=3 / 2,3 \leq \gamma<\infty$, or $\nabla u_{3} \in L^{4 / 3, \infty}$ then $u(x, t)$ is a strong solution on $[0, T)$.

REMARK 1. In [6], He proved the same conclusion under a stronger condition $\nabla u_{3} \in L^{\alpha, \gamma}$ with $2 / \alpha+3 / \gamma=1$.

Before going to sections, we recall the definition of Leray-Hopf weak solutions.

Definition. A measurable vector $u$ is called a Leray-Hopf weak solution to the Navier- Stokes equations (1), if $u$ satisfies the following properties

(i) $u$ is weakly continuous from $[0, T)$ to $L^{2}\left(\mathbb{R}^{3}\right)$.

(ii) $u$ verifies (1) in the sense of distribution, i.e.,

$$
\int_{0}^{T} \int_{\mathbb{R}^{3}}\left(\frac{\partial \phi}{\partial t}+(u \cdot \nabla) \phi\right) u d x d t+\int_{\mathbb{R}^{3}} u_{0} \phi(x, 0) d x=\int_{0}^{T} \int_{\mathbb{R}^{3}} \nabla u: \nabla \phi d x d t
$$


for all $\phi \in C_{0}^{\infty}\left(\mathbb{R}^{3} \times[0, T)\right)$ with $\operatorname{div} \phi=0$.

$$
\int_{0}^{T} \int_{\mathbb{R}^{3}} u \cdot \nabla \phi d x d t=0
$$

for every $\phi \in C_{0}^{\infty}\left(\mathbb{R}^{3} \times[0, T)\right)$.

(iii) The energy inequality, i.e.,

$$
\|u(., t)\|_{L^{2}}^{2}+2 \int_{0}^{t}\|\nabla u(., s)\|_{L^{2}}^{2} d s \leq\left\|u_{0}\right\|_{L^{2}}^{2}, \quad 0 \leq t \leq T .
$$

By a strong solution we mean a weak solution $u$ such that

$$
u \in L^{\infty}\left(0, T ; H^{1}\right) \cap L^{2}\left(0, T ; H^{2}\right)
$$

It is well-known that strong solutions are regular (say, classical) and unique in the class of weak solutions.

The constants are different from section to section.

2. Proof of the Main Theorem. The proof follows from the framework established in [20].

First, we give a very simple interpolation lemma

Lemma 1. Assume that a measurable function $u(x, t) \in L^{\infty, 2}$ and $\nabla u \in L^{2,2}$ on $\left[0, T^{*}\right), T^{*} \leq T$, then $u \in L^{p, q}$ with $p \geq 2,2 \leq q \leq 6$ and $2 / p+3 / q \geq 3 / 2$ for $0 \leq t \leq T^{*}$

$$
\|u\|_{L^{p, q}} \leq C_{1}\|u\|_{L^{\infty, 2}}^{\frac{3}{q}-\frac{1}{2}}\|\nabla u\|_{L^{2,2}}^{\frac{3}{2}-\frac{3}{q}}
$$

where $C_{1}=C_{1}(p, q, T)$. If $\frac{2}{p}+\frac{3}{q}=\frac{3}{2}$, then

$$
\|u\|_{L^{p, q}} \leq C_{1}(q)\|u\|_{L^{\infty, 2}, 1}^{1-\frac{2}{p}}\|\nabla u\|_{L^{2,2}}^{\frac{3}{2}-\frac{3}{q}}
$$

The proof is simple (see Lemma 1 in [21]).

The second lemma is the following Gronwall type inequality.

Lemma 2 [4]. $a(x)$ and $b(x)$ be nonnegative functions on $[0, A)$ and $0<\delta<1$. Suppose a nonnegative function $y(x)$ satisfies the differential inequality

$$
y^{\prime}(x)+b(x) \leq a(x) y^{\delta}(x) \text { on }[0, A), y(0)=y_{0} .
$$

Then for $0 \leq x<A$,

$$
y(x)+\int_{0}^{x} b(s) d s \leq\left(2^{\delta /(1-\delta)}+1\right) y_{0}+2^{\delta /(1-\delta)}\left(\int_{0}^{x} a(s) d s\right)^{1 /(1-\delta)} .
$$

Proof. Solving the homogeneous differential inequality $y^{\prime} \leq a(x) y^{\delta}$, one obtains

$$
y(x) \leq\left\{y_{0}^{1-\delta}+\int_{0}^{x} a(s) d s\right\}^{1 /(1-\delta)} .
$$


substituting (6) into (4) and integrating over $[0, x]$, we obtain

$$
\begin{aligned}
y(x)+\int_{0}^{x} b(s) d s & \leq \int_{0}^{x} a(s) d s\left\{y_{0}^{1-\delta}+\int_{0}^{x} a(s) d s\right\}^{\delta /(1-\delta)}+y_{0} \\
& \leq\left\{y_{0}^{1-\delta}+\int_{0}^{x} a(s) d s\right\}^{1 /(1-\delta)}+y_{0} \\
& \leq 2^{\delta /(1-\delta)}\left\{y_{0}+\left(\int_{0}^{x} a(s) d s\right)^{1 /(1-\delta)}\right\}+y_{0} .
\end{aligned}
$$

This complete the proof.

Now we go to the proof of the main theorem. Since there are some differences between the proof for $\gamma=3$ and $\gamma>3$, we divide the proof into two parts.

Proof of TheOrem 1 FOR $\gamma=3$. Now our condition is that $u$ is a Leray-Hopf weak solution on $(0, T)$ with $\nabla u_{3} \in L^{4,3}$. For the vorticity field $\omega=\operatorname{curl} u=\left(\omega_{1}, \omega_{2}, \omega_{3}\right)$, one has the following estimate.

Lemma 3. Suppose $u_{0} \in H^{1}\left(\mathbb{R}^{3}\right)$ with $\operatorname{div} u_{0}=0$. Assume that $(u, p)$ is a smooth solution in $\mathbb{R}^{3} \times(0, T)$, which satisfies the energy inequality, with $\nabla u \in L^{\infty, 2}$ and $\Delta u \in L^{2,2}$. If $\nabla u_{3} \in L^{4,3}\left(\mathbb{R}^{3} \times(0, T)\right)$, then for $0 \leq t<T$

$$
\begin{gathered}
\left\|\omega_{3}(., t)\right\|_{L^{2}}^{2}+\int_{0}^{t}\left\|\nabla \omega_{3}(., \tau)\right\|_{L^{2}}^{2} d \tau \\
\leq 3\left\|\omega_{3}^{0}\right\|_{L^{2}}^{2}+C_{2}\left\|\nabla u_{3}\right\|_{L^{4,3}}^{2}\|\nabla u\|_{L^{\infty, 2}}^{\frac{1}{2}}\|\Delta u\|_{L^{2,2}}^{\frac{1}{2}}
\end{gathered}
$$

where $C_{2}=C_{2}\left(\left\|u_{0}\right\|_{L^{2}}\right)$ and $\omega^{0}(x)$ is the initial datum for $\omega$.

Proof. Vorticity $\omega=\operatorname{curl} u$ satisfies

$$
\left\{\begin{array}{l}
\frac{\partial \omega}{\partial t}+(u \cdot \nabla) \omega=(\omega \cdot \nabla) u+\Delta \omega \\
\operatorname{div} u=0 \\
\operatorname{curl} u=\omega \\
\omega(x, 0)=\omega^{0}(x)
\end{array}\right.
$$

Multiplying the first equation of (8) by $\omega_{3}$, and integrating on $\mathbb{R}^{3}$, after suitable integration by parts, we obtain

$$
\begin{aligned}
& \frac{1}{2} \frac{d}{d t}\left\|\omega_{3}(., t)\right\|_{L^{2}}^{2}+\left\|\nabla \omega_{3}(., t)\right\|_{L^{2}}^{2} \\
& \leq \int_{\mathbb{R}^{3}}\left|\left(\omega \cdot \nabla u_{3}\right) \omega_{3}\right| d x \\
& \leq 2\|\nabla u\|_{L^{\frac{12}{5}}}\left\|\nabla u_{3}\right\|_{L^{3}}\left\|\omega_{3}\right\|_{L^{4}} \quad(|\omega|<2|\nabla u|) \\
& \leq C_{3}\|\nabla u\|_{L^{\frac{12}{5}}}\left\|\nabla u_{3}\right\|_{L^{3}}\left\|\omega_{3}\right\|_{L^{2}}^{\frac{1}{4}}\left\|\nabla \omega_{3}\right\|_{L^{2}}^{\frac{3}{4}} \quad \text { (Gagliardo-Nirenberg inequality) } \\
& \leq \frac{1}{2}\left\|\nabla \omega_{3}\right\|_{L^{2}}^{2}+C_{3}\|\nabla u\|_{L^{\frac{12}{5}}}^{8 / 5}\left\|\nabla u_{3}\right\|_{L^{3}}^{8 / 5}\left\|\omega_{3}\right\|_{L^{2}}^{2 / 5} \quad \text { (Young iequality). }
\end{aligned}
$$


Then we can apply Lemma 2 on (9) corresponding to $\delta=\frac{1}{5}$ in Lemma 2 ,

$$
\begin{aligned}
& \left\|\omega_{3}(., t)\right\|_{L^{2}}^{2}+\int_{0}^{t}\left\|\nabla \omega_{3}(., \tau)\right\|_{L^{2}}^{2} d \tau \\
& \leq 3\left\|\omega_{3}^{0}\right\|_{L^{2}}^{2}+C_{4}\left\{\int_{0}^{t}\|\nabla u\|_{L^{\frac{12}{5}}}^{8 / 5}\left\|\nabla u_{3}\right\|_{L^{3}}^{8 / 5} d \tau\right\}^{5 / 4} \\
& \leq 3\left\|\omega_{3}^{0}\right\|_{L^{2}}^{2}+C_{4}\left\{\int_{0}^{t}\|\nabla u\|_{L^{2}}^{4 / 5}\|\nabla u\|_{L^{3}}^{4 / 5}\left\|\nabla u_{3}\right\|_{L^{3}}^{8 / 5} d \tau\right\}^{5 / 4} \\
& \leq 3\left\|\omega_{3}^{0}\right\|_{L^{2}}^{2}+C_{4}\|\nabla u\|_{L^{2,2}}\|\nabla u\|_{L^{4,3}}\left\|\nabla u_{3}\right\|_{L^{4,3}}^{2} \\
& \leq 3\left\|\omega_{3}^{0}\right\|_{L^{2}}^{2}+C_{5}\|\nabla u\|_{L^{\infty, 2}}^{\frac{1}{2}}\|\Delta u\|_{L^{2,2}}^{\frac{1}{2}}\left\|\nabla u_{3}\right\|_{L^{4,3}}^{2}
\end{aligned}
$$

where we use the energy inequality and apply Lemma 1 on $\|\nabla u\|_{L^{4,3}}$, since $\frac{2}{4}+\frac{3}{3}=\frac{3}{2}$. So we finish the proof.

After the a priori estimate on $\omega_{3}$, we establish the following a priori estimate for the velocity field.

LEMMA 4. Under the same condition as that in Lemma 3, we have

$$
\sup _{0 \leq t<T}\|\nabla u(., t)\|_{L^{2}}^{2}+\int_{0}^{T}\|\Delta u(., \tau)\|_{L^{2}}^{2} d \tau \leq C_{6}
$$

where $C_{6}$ depends on $T,\left\|\nabla u_{0}\right\|_{L^{2}},\left\|\nabla u_{0}\right\|_{L^{2}}$ and $\left\|\nabla u_{3}\right\|_{L^{4,3}}$.

Proof. As we have done in [21] we can rewrite the first equation of the NavierStokes equations (1) as

$$
\frac{\partial u}{\partial t}+\omega \times u+\frac{1}{2} \nabla|u|^{2}+\nabla p=\Delta u
$$

Multiply the equation (11) by $\Delta u$ and integrate on $\mathbb{R}^{3} \times(0, t)$, after suitable integration by parts, one obtains

$$
\begin{aligned}
& \frac{1}{2}\|\nabla u(., t)\|_{L^{2}}^{2}+\int_{0}^{t}\|\Delta u(., \tau)\|_{L^{2}}^{2} d \tau \\
= & \int_{0}^{t} \int_{\mathbb{R}^{3}}(\omega \times u) \cdot \Delta u d x d \tau+\frac{1}{2}\left\|\nabla u_{0}\right\|_{L^{2}}^{2}
\end{aligned}
$$

let

$$
\begin{aligned}
I= & \int_{0}^{t} \int_{\mathbb{R}^{3}}(\omega \times u) \cdot \Delta u d x d \tau \\
\leq & \int_{0}^{t} \int_{\mathbb{R}^{3}}\left|\omega_{2} u_{3} \Delta u_{1}\right| d x d \tau+\int_{0}^{t} \int_{\mathbb{R}^{3}}\left|\omega_{3} u_{2} \Delta u_{1}\right| d x d \tau+\int_{0}^{t} \int_{\mathbb{R}^{3}}\left|\omega_{3} u_{1} \Delta u_{2}\right| d x d \tau \\
& +\int_{0}^{t} \int_{\mathbb{R}^{3}}\left|\omega_{2} u_{3} \Delta u_{2}\right| d x d \tau+\left|\int_{0}^{t} \int_{\mathbb{R}^{3}} \omega_{1} u_{2} \Delta u_{3} d x d \tau\right|+\left|\int_{0}^{t} \int_{\mathbb{R}^{3}} \omega_{2} u_{1} \Delta u_{3} d x d \tau\right| \\
\equiv & I_{1}+I_{2}+I_{3}+I_{4}+I_{5}+I_{6}
\end{aligned}
$$


We will estimate the terms one by one.

$$
\begin{aligned}
I_{1}= & \int_{0}^{t} \int_{\mathbb{R}^{3}}\left|\omega_{2} u_{3} \Delta u_{1}\right| d x d \tau \\
\leq & \int_{0}^{t}\left\|\omega_{2}\right\| L_{L^{4}}\left\|u_{3}\right\|_{L^{4}}\|\Delta u\|_{L^{2}} d \tau \\
\leq & C_{7} \int_{0}^{t}\|\nabla u\|_{L^{2}}^{1 / 4}\left\|\nabla u_{3}\right\|_{L^{3}}^{1 / 2}\|u\|_{L^{2}}^{1 / 2}\|\Delta u\|_{L^{2}}^{7 / 4} d \tau \\
& \left(\text { Gagliardo-Nirenberg inequality, for } \omega_{2} \text { and } u_{3}\right) \\
\leq & \frac{1}{20}\|\Delta\|_{L^{2,2}}^{2}+C_{8}\left\|\nabla u_{3}\right\|_{L^{4,3}}^{4}\|\nabla u\|_{L^{\infty, 2}}^{2}, \quad \text { (Young inequality) }
\end{aligned}
$$

where $C_{8}$ is a constant depending on $\left\|u_{0}\right\|_{L^{2}}$ only.

$$
\begin{aligned}
I_{2} \leq & \frac{1}{20}\|\Delta u\|_{L^{2,2}}^{2}+5 \int_{0}^{t}\left\|u_{2}\right\|_{L^{a}}^{2}\left\|\omega_{3}\right\|_{L^{b}}^{2} d \tau \\
& \left(\text { Hölder's and Young inequality } \frac{1}{a}+\frac{1}{b}=\frac{1}{2}\right) \\
\leq & \left.\frac{1}{20}\|\Delta u\|_{L^{2,2}}^{2}+5\left\|u_{2}\right\|_{L^{p, a}}^{2}\left\|\omega_{3}\right\|_{L^{q, b}}^{2} \quad \text { (Hölder's inequality } \frac{1}{p}+\frac{1}{q}=\frac{1}{2}\right)
\end{aligned}
$$

Now we want to apply Lemma 1 on $\left\|w_{3}\right\|_{L^{q, b}}$, so $a, b, p$ and $q$ satisfies

$$
\left\{\begin{array}{l}
\frac{1}{a}+\frac{1}{b}=\frac{1}{2} \\
\frac{1}{p}+\frac{1}{q}=\frac{1}{2} \\
\frac{2}{q}+\frac{3}{b}=\frac{3}{2}
\end{array}\right.
$$

(14) can be solved as

$$
\left\{\begin{array}{l}
p=\infty, \quad a=3 \\
q=2, \quad b=6
\end{array}\right.
$$

Then Lemma 3 tells us

$$
\left\|\omega_{3}\right\|_{L^{2,6}}^{2} \leq C_{9}\left\|\nabla u_{3}\right\|_{L^{4,3}}^{2}\|\nabla u\|_{L^{\infty, 2}}^{1 / 2}\|\Delta u\|_{L^{2,2}}^{1 / 2}+C_{10}
$$

where $C_{9}$ and $C_{10}$ are constants depending only on $\left\|\omega_{3}^{0}\right\|_{L^{2}}$.

On the other hand,

$$
\begin{aligned}
\left\|u_{2}\right\|_{L^{\infty, 3}}^{2} & \leq\|u\|_{L^{\infty, 3}}^{2} \\
& \leq\|u\|_{L^{\infty, 2}}\|u\|_{L^{\infty, 6}} \\
& \leq C_{11}\|\nabla u\|_{L^{\infty, 2}}, \quad \text { (Energy inequality and Sobolev inequality) }
\end{aligned}
$$

where $C_{11}$ depends on $\left\|u_{0}\right\|_{L^{2}}$ only.

Therefore $I_{2}$ can be estimated as

$$
I_{2} \leq \frac{1}{20}\|\Delta u\|_{L^{2,2}}^{2}+C_{12}\|\nabla u\|_{L^{\infty, 2}}^{3 / 2}\|\Delta u\|_{L^{2,2}}^{1 / 2}\left\|\nabla u_{3}\right\|_{L^{4,3}}^{2}+C_{13}\|\nabla u\|_{L^{\infty, 2}},
$$

where $C_{12}$ depends on $\left\|u_{0}\right\|_{L^{2}}$, while $C_{13}$ depends on $\left\|u_{0}\right\|_{L^{2}}$ and $\left\|\omega_{3}^{0}\right\|_{L^{2}}$. 
$I_{3}$ is similar to $I_{2}$,

$$
I_{3} \leq \frac{1}{20}\|\Delta u\|_{L^{2,2}}^{2}+C_{12}\|\nabla u\|_{L^{\infty, 2}}^{3 / 2}\|\Delta u\|_{L^{2,2}}^{1 / 2}\left\|\nabla u_{3}\right\|_{L^{4,3}}^{2}+C_{13}\|\nabla u\|_{L^{\infty, 2},},
$$

and $I_{4}$ is similar to $I_{1}$,

$$
\begin{gathered}
I_{4} \leq \frac{1}{20}\|\Delta u\|_{L^{2,2}}^{2}+C_{8}\left\|\nabla u_{3}\right\|_{L^{4,3}}^{4}\|\nabla u\|_{L^{\infty, 2}}^{2} \\
I_{5}=\left|\int_{0}^{t} \int_{\mathbb{R}^{3}} \omega_{1} u_{2} \Delta u_{3} d x d \tau\right| \\
\leq \int_{0}^{t} \int_{\mathbb{R}^{3}}\left|\left(\partial_{2} u_{3}\right) u_{2} \Delta u_{3}\right| d x d \tau+\left|\int_{0}^{t} \int_{\mathbb{R}^{3}}\left(\partial_{3} u_{2}\right) u_{2} \Delta u_{3} d x d \tau\right| \equiv I_{5}^{1}+I_{5}^{2} \\
I_{5}^{1}=\int_{0}^{t} \int_{\mathbb{R}^{3}}\left|\left(\partial_{2} u_{3}\right) u_{2} \Delta u_{3}\right| d x d \tau \\
\leq C_{14} \int_{0}^{t}\|\Delta u\|_{L^{2}}\left\|\nabla u_{3}\right\|_{L^{3}}\|\nabla u\|_{L^{2}} d \tau \text { (Hölder's and Sobolev inequality) } \\
\leq \frac{1}{40}\|\Delta u\|_{L^{2,2}}^{2}+5 C_{14} T^{1 / 2}\left\|\nabla u_{3}\right\|_{L^{4,3}}^{2}\|\nabla u\|_{L^{\infty, 2}}^{2},
\end{gathered}
$$

where in the last inequality, we use Young and Hölder's inequality. For simplicity, we denote $5 C_{14} T^{1 / 2}$ as $C_{15}$ which depends on $T$.

$$
\begin{aligned}
I_{5}^{2}= & \left|\int_{0}^{t} \int_{\mathbb{R}^{3}}\left(\partial_{3} u_{3}\right) u_{2} \Delta u_{3} d x d \tau\right|=\left|\int_{0}^{t} \int_{\mathbb{R}^{3}} \frac{1}{2} u_{2}^{2} \Delta\left(\partial_{3} u_{3}\right) d x d \tau\right| \\
\leq & \left|\int_{0}^{t} \int_{\mathbb{R}^{3}}\left(\partial_{3} u_{3}\right) u_{2} \Delta u_{2} d x d \tau\right|+\left.\left|\int_{0}^{t} \int_{\mathbb{R}^{3}}\left(\partial_{3} u_{3}\right)\right| \nabla u_{2}\right|^{2} d x d \tau \mid \\
\leq & \frac{1}{40}\|\Delta u\|_{L^{2,2}}^{2}+C_{15}\left\|\nabla u_{3}\right\|_{L^{4,3}}^{2}\|\nabla u\|_{L^{\infty, 2}}^{2}+\int_{0}^{t}\left\|\nabla u_{2}\right\|_{L^{3}}^{2}\left\|\nabla u_{3}\right\|_{L^{3}} d \tau \\
& (\text { By }(20) \text { and Hölder's inequality respectively) } \\
\leq & \frac{1}{40}\|\Delta u\|_{L^{2,2}}^{2}+C_{15}\left\|\nabla u_{3}\right\|_{L^{4,3}}^{2}\|\nabla u\|_{L^{\infty, 2}}^{2}+C_{16} \int_{0}^{t}\|\nabla u\|_{L^{2}}\|\Delta u\|_{L^{2}}\left\|\nabla u_{3}\right\|_{L^{3}} d \tau \\
\leq & \frac{1}{20}\|\Delta u\|_{L^{2,2}}^{2}+C_{17}\left\|\nabla u_{3}\right\|_{L^{4,3}}^{2}\|\nabla u\|_{L^{\infty, 2}}^{2}
\end{aligned}
$$

$I_{6}$ can be treated similarly

$$
I_{6} \leq \frac{3}{40}\|\Delta u\|_{L^{2,2}}^{2}+\left(C_{15}+C_{17}\right)\left\|\nabla u_{3}\right\|_{L^{4,3}}^{2}\|\nabla u\|_{L^{\infty, 2}}^{2}
$$

where $C_{15}$ and $C_{17}$ depend only on $T$.

Substituting the above estimates (13), (17), (18), (19), (20), (21) and (22) into (12), it follows that

$$
\begin{aligned}
& \frac{1}{2}\|\nabla u(., t)\|_{L^{2}}^{2}+\frac{13}{20}\|\Delta u\|_{L^{2,2}}^{2}-\frac{1}{2}\left\|\nabla u_{0}\right\|_{L^{2}}^{2} \\
& \leq 2\left(C_{8}\left\|\nabla u_{3}\right\|_{L^{4,3}}^{4}+\left(C_{15}+C_{17}\right)\left\|\nabla u_{3}\right\|_{L^{4,3}}^{2}\right)\|\nabla u\|_{L^{\infty, 2}}^{2} \\
& \quad+2 C_{12}\|\nabla u\|_{L^{\infty, 2}}^{3 / 2}\|\Delta u\|_{L^{2,2}}^{1 / 2}\left\|\nabla u_{3}\right\|_{L^{4,3}}^{2}+2 C_{13}\|\nabla u\|_{L^{\infty, 2}} \\
& \leq 2\left(C_{8}\left\|\nabla u_{3}\right\|_{L^{4,3}}^{4}+\left(C_{15}+C_{17}\right)\left\|\nabla u_{3}\right\|_{L^{4,3}}^{2}\right)\|\nabla u\|_{L^{\infty, 2}}^{2} \\
& \quad+4 C_{18}\left\|\nabla u_{3}\right\|_{L^{4,3}}^{8 / 3}\|\nabla u\|_{L^{\infty, 2}}^{2}+\frac{3}{20}\|\Delta u\|_{L^{2,2}}^{2}+4 C_{13}^{2}+\frac{1}{8}\|u\|_{L^{\infty, 2}}^{2}
\end{aligned}
$$


where $C_{18}$ is a constant depends on $\left\|u_{0}\right\|_{L^{2}}$. Hence

$$
\begin{aligned}
& \|\nabla u(., t)\|_{L^{2}}^{2}+\|\Delta u\|_{L^{2,2}}-\left\|\nabla u_{0}\right\|_{L^{2}}^{2} \\
& \leq 4\left(C_{8}\left\|\nabla u_{3}\right\|_{L^{4,3}}^{4}+\left(C_{15}+C_{17}\right)\left\|\nabla u_{3}\right\|_{L^{4,3}}^{2}\right. \\
& \left.\quad+2 C_{18}\left\|\nabla u_{3}\right\|_{L^{4,3}}^{8 / 3}\right)\|\nabla u\|_{L^{\infty, 2}}^{2}+8 C_{13}^{2}+\frac{1}{4}\|u\|_{L^{\infty, 2}}^{2},
\end{aligned}
$$

Now we choose $0<t_{0} \leq T$, which is small enough, such that

$$
\begin{aligned}
& C_{8} \int_{0}^{t_{0}}\left\|\nabla u_{3}(., \tau)\right\|_{L^{3}}^{4} d \tau+\left(C_{15}+C_{17}\right)\left(\int_{0}^{t_{0}}\left\|\nabla u_{3}(., \tau)\right\|_{L^{3}}^{4} d \tau\right)^{1 / 2} \\
& +2 C_{18}\left(\int_{0}^{t_{0}}\left\|\nabla u_{3}(., \tau)\right\|_{L^{3}}^{4}\right)^{2 / 3} \leq \frac{1}{8}
\end{aligned}
$$

and consequently from (23), we obtain that

$$
\sup _{0 \leq t \leq t_{0}}\|\nabla u(., t)\|_{L^{2}}^{2}+\int_{0}^{t_{0}}\|\Delta u(., \tau)\|_{L^{2}}^{2} d \tau \leq 32 C_{13}^{2}+4\left\|\nabla u_{0}\right\|_{L^{2}}^{2} .
$$

Then we can repeat the above process from $t_{0}$, if $t_{0}<T$, with $u\left(t_{0}\right)$ as its initial data for the problem (1) and obtain a similar estimate as (23), for $t_{0} \leq t<T$,

$$
\begin{aligned}
& \|\nabla u(., t)\|_{L^{2}}^{2}+\int_{t_{0}}^{t}\|\Delta u(., \tau)\|_{L^{2}}^{2} d \tau \\
& \leq 4\left(C_{8}\left\|\nabla u_{3}\right\|_{L^{4,3}}^{4}+\left(C_{15}+C_{17}\right)\left\|\nabla u_{3}\right\|_{L^{4,3}}^{2}+2 C_{18}\left\|\nabla u_{3}\right\|_{L^{4,3}}^{8 / 3}\right) \sup _{t_{0} \leq \tau \leq t}\|\nabla u\|_{L^{2}}^{2} \\
& \quad+8 C_{13}^{2}+\frac{1}{4} \sup _{t_{0} \leq \tau \leq t}\|u\|_{L^{2}}^{2}+\left\|\nabla u\left(., t_{0}\right)\right\|_{L^{2}}^{2}
\end{aligned}
$$

There exists a number for $t_{1}$, such that

$$
\begin{aligned}
& C_{8} \int_{t_{0}}^{t_{1}}\left\|\nabla u_{3}(., \tau)\right\|_{L^{3}}^{4} d \tau+\left(C_{15}+C_{17}\right)\left(\int_{t_{0}}^{t_{1}}\left\|\nabla u_{3}(., \tau)\right\|_{L^{3}}^{4} d \tau\right)^{1 / 2} \\
& +2 C_{18}\left(\int_{t_{0}}^{t_{1}}\left\|\nabla u_{3}(., \tau)\right\|_{L^{3}}^{4}\right)^{2 / 3} \leq \frac{1}{8}
\end{aligned}
$$

therefore we have

$$
\begin{aligned}
& \sup _{t_{0} \leq t \leq t_{1}}\|\nabla u(., t)\|_{L^{2}}^{2}+\int_{t_{0}}^{t_{1}}\|\Delta u(., \tau)\|_{L^{2}}^{2} d \tau \\
& \leq 32 C_{13}^{2}+4\left\|\nabla u\left(., t_{0}\right)\right\|_{L^{2}}^{2} \leq 96 C_{13}^{2}+8\left\|\nabla u\left(., t_{0}\right)\right\|_{L^{2}}^{2}
\end{aligned}
$$

Then we can repeat the above process from $t_{1}$, if $t_{1}<T$. Actually, since $\nabla u_{3} \in L^{4,3}$ on $[0, T)$, and the coefficients involving $\left\|u_{3}\right\|_{L^{4,3}}$ in (23), which depend only on $T, p$, $b,\left\|u_{0}\right\|_{L^{2}}$, the above process only can be done for finite times. More precisely, we can get a estimate on the whole time interval.

$$
\sup _{0 \leq t<T}\|\nabla u(., t)\|_{L^{2}}^{2}+\int_{0}^{T}\|\Delta u(., \tau)\|_{L^{2}}^{2} d \tau \leq C_{6}
$$


where $C_{6}$ depends on $T,\left\|\nabla u_{0}\right\|_{L^{2}},\left\|u_{0}\right\|_{L^{2}}$ and $\left\|u_{3}\right\|_{L^{4,3}}$. $\mathrm{Z}$

After we got the a priori estimate, the proof of Theorem 1 for the case $\gamma=3$ is simple. It is well known [19] that there is a unique strong solution $\tilde{u} \in L^{\infty}\left(0, T_{0} ; H^{1}\left(\mathbb{R}^{3}\right)\right) \cap u \in$ $L^{2}\left(0, T_{0} ; H^{2}\left(\mathbb{R}^{3}\right)\right)$ to $(1)$,for some $0<T_{0}$, for any given $u_{0} \in L^{2}\left(\mathbb{R}^{3}\right) \cap H^{1}\left(\mathbb{R}^{3}\right)$ with $\operatorname{div} u_{0}=0$. Since $u$ is a Leray-Hopf weak solution which satisfies the energy inequality, we have according to the uniqueness result, $u \equiv \tilde{u}$ on $\left[0, T_{0}\right)$. By the a priori estimate ( 25 ) and standard continuation argument, the local strong solution $u$ can be extended to time $T$. So we have proved $u$ actually is a strong solution on $[0, T)$.

Proof of TheOrem 1 FOR $\gamma>3$. Like the proof for $\gamma=3$, we want to give an estimate on $\omega_{3}$ first. The constants are different from the above's.

Lemma 5. Suppose $u_{0} \in H^{1}\left(\mathbb{R}^{3}\right)$ with $\operatorname{div} u_{0}=0$. Assume that $(u, p)$ is a smooth solution in $\mathbb{R}^{3} \times(0, T)$, which satisfies the energy inequality, with $\nabla u \in L^{\infty, 2}$ and $\Delta u \in L^{2,2}$. If $\nabla u_{3} \in L^{\alpha, \gamma}\left(\mathbb{R}^{3} \times(0, T)\right)$ for $\frac{2}{\alpha}+\frac{3}{\gamma} \leq \frac{3}{2}$, then for $0 \leq t \leq T^{*}$

$$
\begin{gathered}
\left\|\omega_{3}(., t)\right\|_{L^{2}}^{2}+\int_{0}^{t}\left\|\nabla \omega_{3}(., \tau)\right\|_{L^{2}}^{2} d \tau \\
\leq 3\left\|\omega_{3}^{0}\right\|_{L^{2}}^{2}+C_{2}\left\|\nabla u_{3}\right\|_{L^{\alpha, \gamma}}^{2}\|\nabla u\|_{L^{\infty, 2}}^{1-\frac{3}{\gamma}}\|\Delta u\|_{L^{2,2}}^{\frac{3}{\gamma}}
\end{gathered}
$$

where $C_{2}=C_{2}\left(\gamma,\left\|u_{0}\right\|_{L^{2}}\right)$ and $\omega^{0}(x)$ is the initial datum for $\omega$.

Proof. The proof is more difficult than that of Lemma 3, although the method is same. You know, when you use Hölder's inequality, how to choose the numbers which are suitable for the estimates is difficult since there are so many choices.

Multiplying the first equation of (8) by $\omega_{3}$, and integrating on $\mathbb{R}^{3}$, after suitable integration by parts, we obtain

$$
\begin{aligned}
& \frac{1}{2} \frac{d}{d t}\left\|\omega_{3}(., t)\right\|_{L^{2}}^{2}+\left\|\nabla \omega_{3}(., t)\right\|_{L^{2}}^{2} \\
& \leq \int_{\mathbb{R}^{3}}\left|\left(\omega \cdot \nabla u_{3}\right) \omega_{3}\right| d x \\
& \leq 2\|\nabla u\|_{L^{\frac{2 \gamma}{\gamma-1}}\left\|\nabla u_{3}\right\|_{L^{\gamma}}\left\|\omega_{3}\right\|_{L^{\frac{2 \gamma}{\gamma-1}}}(|\omega|<2|\nabla u|)} \leq C_{3}\|\nabla u\|_{L^{\frac{2 \gamma}{\gamma-1}}}\left\|\nabla u_{3}\right\|_{L^{\gamma}}\left\|\omega_{3}\right\|_{L^{2}}^{1-\frac{3}{2 \gamma}}\left\|\nabla \omega_{3}\right\|_{L^{2}}^{\frac{3}{2 \gamma}} \text { (Gagliardo-Nirenberg inequality) } \\
& \leq \frac{1}{2}\left\|\nabla \omega_{3}\right\|_{L^{2}}^{2}+C_{3}\|\nabla u\|_{L^{\frac{2 \gamma}{\gamma-1}}}^{\frac{4 \gamma}{4 \gamma-3}}\left\|\nabla u_{3}\right\|_{L^{\gamma}-3}^{\frac{4 \gamma}{4 \gamma-3}}\left\|\omega_{3}\right\|_{L^{2}}^{\frac{(2 \gamma-3) 2}{4 \gamma-3}} \quad \text { (Young iequality). }
\end{aligned}
$$


Then we can apply Lemma 2 on (27) corresponding to $\delta=\frac{2 \gamma-3}{4 \gamma-3}$ in Lemma 2,

$$
\begin{aligned}
& \left\|\omega_{3}(., t)\right\|_{L^{2}}^{2}+\int_{0}^{t}\left\|\nabla \omega_{3}(., \tau)\right\|_{L^{2}}^{2} d \tau \\
& \leq 3\left\|\omega_{3}^{0}\right\|_{L^{2}}^{2}+C_{4}\left\{\int_{0}^{t}\|\nabla u\|_{L^{\frac{2 \gamma}{\gamma-1}}}^{\frac{4 \gamma}{4 \gamma-3}}\left\|\nabla u_{3}\right\|_{L^{\gamma}-3}^{\frac{4 \gamma}{4 \gamma-3}} d \tau\right\}^{\frac{4 \gamma-3}{2 \gamma}} \\
& \leq 3\left\|\omega_{3}^{0}\right\|_{L^{2}}^{2}+C_{4}\left\{\int_{0}^{t}\|\nabla u\|_{L^{2}}^{\frac{2 \gamma}{4 \gamma-3}}\|\nabla u\|_{L^{\frac{2 \gamma}{\gamma-2}}}^{\frac{2 \gamma}{4 \gamma-3}}\left\|\nabla u_{3}\right\|_{L^{\gamma}}^{\frac{4 \gamma}{4 \gamma-3}} d \tau\right\}^{\frac{4 \gamma-3}{2 \gamma}}
\end{aligned}
$$

(Interpolation inequality $\frac{\gamma-1}{2 \gamma}=\frac{1}{2} \frac{1}{2}+\frac{1}{2} \frac{\gamma-2}{2 \gamma}$ )

$\leq 3\left\|\omega_{3}^{0}\right\|_{L^{2}}^{2}+C_{4}\|\nabla u\|_{L^{\infty, 2}}\|\nabla u\|_{L^{\frac{2 \gamma}{3}, \frac{2 \gamma}{\gamma-2}}}\left\|\nabla u_{3}\right\|_{L^{\alpha, \gamma}}^{2}$

(Hölder's inequality $\frac{\frac{2 \gamma}{4 \gamma-3}}{2}+\frac{\frac{4 \gamma}{4 \gamma-3}}{\alpha}+\frac{\frac{2 \gamma}{4 \gamma-3}}{2 \gamma / 3}=1$ )

$\leq 3\left\|\omega_{3}^{0}\right\|_{L^{2}}^{2}+C_{5}\|\nabla u\|_{L^{\infty, 2}}^{1-\frac{3}{\gamma}}\|\Delta u\|_{L^{2,2}}^{\frac{3}{\gamma}}\left\|\nabla u_{3}\right\|_{L^{\alpha, \gamma}}^{2}$

(Energy inequality and Lemma 1 since $\frac{2}{2 \gamma / 3}+\frac{3}{\frac{2 \gamma}{\gamma-2}}=\frac{3}{2}$ )

The proof is complete.

LEMma 6. Under the same condition as that in Lemma 5, we have

$$
\sup _{0 \leq t<T}\|\nabla u(., t)\|_{L^{2}}^{2}+\int_{0}^{T}\|\Delta u(., \tau)\|_{L^{2}}^{2} d \tau \leq C_{6}
$$

where $C_{6}$ depends on $\alpha, \gamma,\left\|\nabla u_{0}\right\|_{L^{2}},\left\|u_{0}\right\|_{L^{2}}$ and $\left\|u_{3}\right\|_{L^{\alpha, \gamma}}$.

Proof.Rewrite the first equation of the Navier-Stokes equations (1) as

$$
\frac{\partial u}{\partial t}+\omega \times u+\frac{1}{2} \nabla|u|^{2}+\nabla p=\Delta u .
$$

Multiply the equation (29) by $\Delta u$ and integrate on $\mathbb{R}^{3} \times(0, t)$, after suitable integration by parts, one obtains

$$
\begin{aligned}
& \frac{1}{2}\|\nabla u(., t)\|_{L^{2}}^{2}+\int_{0}^{t}\|\Delta u(., \tau)\|_{L^{2}}^{2} d \tau \\
= & \int_{0}^{t} \int_{\mathbb{R}^{3}}(\omega \times u) \cdot \Delta u d x d \tau+\frac{1}{2}\left\|\nabla u_{0}\right\|_{L^{2}}^{2}
\end{aligned}
$$

let

$$
\begin{aligned}
I= & \int_{0}^{t} \int_{\mathbb{R}^{3}}(\omega \times u) \cdot \Delta u d x d \tau \\
\leq & \int_{0}^{t} \int_{\mathbb{R}^{3}}\left|\omega_{2} u_{3} \Delta u_{1}\right| d x d \tau+\int_{0}^{t} \int_{\mathbb{R}^{3}}\left|\omega_{3} u_{2} \Delta u_{1}\right| d x d \tau+\int_{0}^{t} \int_{\mathbb{R}^{3}}\left|\omega_{3} u_{1} \Delta u_{2}\right| d x d \tau \\
& +\int_{0}^{t} \int_{\mathbb{R}^{3}}\left|\omega_{1} u_{3} \Delta u_{2}\right| d x d \tau+\left|\int_{0}^{t} \int_{\mathbb{R}^{3}} \omega_{1} u_{2} \Delta u_{3} d x d \tau\right|+\left|\int_{0}^{t} \int_{\mathbb{R}^{3}} \omega_{2} u_{1} \Delta u_{3} d x d \tau\right| \\
\equiv & I_{1}+I_{2}+I_{3}+I_{4}+I_{5}+I_{6}
\end{aligned}
$$


We will estimate the terms one by one.

$$
\begin{aligned}
& I_{1}=\int_{0}^{t} \int_{\mathbb{R}^{3}}\left|\omega_{2} u_{3} \Delta u_{1}\right| d x d \tau \\
& \leq \int_{0}^{t}\left\|\omega_{2}\right\|_{L^{\frac{6 \alpha}{\alpha+2}}}\left\|u_{3}\right\|_{L^{\frac{3 \alpha}{\alpha-1}}}\|\Delta u\|_{L^{2}} d \tau \\
& \leq C_{7} \int_{0}^{t}\|\nabla u\|_{L^{2}}^{1 / \alpha}\left\|\nabla u_{3}\right\|_{L^{\gamma}}^{1 / 2}\|u\|_{L^{2}}^{1 / 2}\|\Delta u\|_{L^{2}}^{2-1 / \alpha} d \tau \\
&\left(\text { Gagliardo-Nirenberg inequality, for } \omega_{2} \text { and } u_{3}\right) \\
& \leq \frac{1}{20}\|\Delta u\|_{L^{2,2}}^{2}+C_{8}\left\|u_{3}\right\|_{L^{\alpha, \gamma}}^{\alpha}\|\nabla u\|_{L^{\infty, 2}}^{2} \quad \text { (Young inequality) } \\
& I_{2} \leq \frac{1}{20}\|\Delta u\|_{L^{2,2}}^{2}+5 \int_{0}^{t}\left\|u_{2}\right\|_{L^{a}}^{2}\left\|\omega_{3}\right\|_{L^{b}}^{2} d \tau \\
&\left(\text { Hölder's and Young inequality } \frac{1}{a}+\frac{1}{b}=\frac{1}{2}\right) \\
& \leq \frac{1}{20}\|\Delta u\|_{L^{2,2}}^{2}+5\left\|u_{2}\right\|_{L^{p, a}}^{2}\left\|\omega_{3}\right\|_{L^{q, b}}^{2} \quad\left(\text { Hölder's inequality } \frac{1}{p}+\frac{1}{q}=\frac{1}{2}\right)
\end{aligned}
$$

Just as the estimate of $I_{2}$ for $\nabla u_{3} \in L^{4,3}$, we can solve $p, q, a$ and $b$ with

$$
\left\{\begin{array}{l}
p=\infty, \quad a=3 \\
q=2, \quad b=6
\end{array}\right.
$$

Then Lemma 5 tells us

$$
\left\|\omega_{3}\right\|_{L^{2,6}} \leq C_{9}\left\|\nabla u_{3}\right\|_{L^{\alpha, \gamma}}\|\nabla u\|_{L^{\infty, 2}}^{1 / 2-\frac{3}{2 \gamma}}\|\Delta u\|_{L^{2,2}}^{\frac{3}{2 \gamma}}+C_{10}
$$

where $C_{9}$ depends on $\gamma$ and $\left\|u_{0}\right\|_{L^{2}}$ and $C_{10}$ depends on $\gamma$ and $\left\|\omega_{3}^{0}\right\|_{L^{2}}$.

On the other hand,

$$
\begin{aligned}
\left\|u_{2}\right\|_{L^{\infty, 3}}^{2} & \leq\|u\|_{L^{\infty, 3}}^{2} \leq\|u\|_{L^{\infty, 2}}\|u\|_{L^{\infty, 6}} \\
& \leq C_{11}\|\nabla u\|_{L^{\infty, 2}} \quad \text { (Energy inequality and Sobolev inequality) }
\end{aligned}
$$

So we have the estimate for $I_{2}$ as

$$
I_{2} \leq C_{12}\|\nabla u\|_{L^{\infty, 2}}^{2-3 / \gamma}\|\Delta u\|_{L^{2,2}}^{3 / \gamma}\left\|\nabla u_{3}\right\|_{L^{\alpha, \gamma}}^{2}+\frac{1}{20}\|\Delta u\|_{L^{2,2}}^{2}+C_{13}\|\nabla u\|_{L^{\infty, 2}},
$$

where $C_{12}$ depends on $\gamma$ and $\left\|u_{0}\right\|_{L^{2}}$, while $C_{13}$ depends on $\gamma,\left\|u_{0}\right\|_{L^{2}}$ and $\left\|\omega_{3}^{0}\right\|_{L^{2}}$.

$I_{3}$ is similar to $I_{2}$,

$$
I_{3} \leq C_{12}\|\nabla u\|_{L^{\infty, 2}}^{2-3 / \gamma}\|\Delta u\|_{L^{2,2}}^{3 / \gamma}\left\|\nabla u_{3}\right\|_{L^{\alpha, \gamma}}^{2}+\frac{1}{20}\|\Delta u\|_{L^{2,2}}^{2}+C_{13}\|\nabla u\|_{L^{\infty, 2}}
$$

and $I_{4}$ is similar to $I_{1}$,

$$
\begin{gathered}
I_{4} \leq \frac{1}{20}\|\Delta u\|_{L^{2,2}}^{2}+C_{8}\left\|u_{3}\right\|_{L^{\alpha, \gamma}}^{\alpha}\|\nabla u\|_{L^{\infty, 2},}^{2} . \\
I_{5}=\left|\int_{0}^{t} \int_{\mathbb{R}^{3}} \omega_{1} u_{2} \Delta u_{3} d x d \tau\right| \\
\leq \int_{0}^{t} \int_{\mathbb{R}^{3}}\left|\left(\partial_{2} u_{3}\right) u_{2} \Delta u_{3}\right| d x d \tau+\left|\int_{0}^{t} \int_{\mathbb{R}^{3}}\left(\partial_{3} u_{2}\right) u_{2} \Delta u_{3} d x d \tau\right| \equiv I_{5}^{1}+I_{5}^{2}
\end{gathered}
$$


Since $2 / \alpha+3 / \gamma=3 / 2$, if $3<\gamma<\infty$, then $4 / 3<\alpha<4$. However, the techniques are different between $4 / 3<\alpha<2$ and $2 \leq \alpha<4$. We deal with $2<\alpha<4$ first.

$$
\begin{aligned}
I_{5}^{1} & =\int_{0}^{t} \int_{\mathbb{R}^{3}}\left|\left(\partial_{2} u_{3}\right) u_{2} \Delta u_{3}\right| d x d \tau \\
& \leq \int_{0}^{t}\|\Delta u\|_{L^{2}}\left\|\nabla u_{3}\right\|_{L^{\gamma}}\left\|u_{2}\right\|_{L^{t}} d \tau \\
& \leq \frac{1}{20}\|\Delta u\|_{L^{2,2}}^{2}+C_{14}\left\|\nabla u_{3}\right\|_{L^{\alpha, \gamma}}^{2}\|u\|_{L^{\frac{2 \alpha}{\alpha-2}, \frac{2 \gamma}{\gamma-2}}}^{2},
\end{aligned}
$$

where we used Hölder's and Young inequality.

On the other hand,

$$
\begin{aligned}
& \|u\|_{L^{\frac{2 \alpha}{\alpha-2}, \frac{2 \gamma}{\gamma-2}}} \leq\|u\|_{L^{\frac{2 \alpha}{\alpha-2}, \frac{6 \alpha}{\alpha+4}}}^{(1-\alpha / 4)}\|u\|_{L^{\frac{2 \alpha}{\alpha-2}, 6}}^{\alpha / 4} \\
& \leq C\left(\left\|u_{0}\right\|_{L^{2}}\right)\|u\|_{L^{\frac{2 \alpha}{\alpha-2}, 6}}^{\alpha / 4} \quad\left(\text { By Lemma } 1 \frac{2}{\frac{2 \alpha}{\alpha-2}}+\frac{3}{\frac{6 \alpha}{\alpha+4}}=\frac{3}{2}\right) \\
& \leq C_{15}\|\nabla u\|_{L^{\infty, 2}}^{1 / 2}\|\nabla u\|_{L^{2,2}}^{\alpha / 4-1 / 2} \\
& \text { (Sobolev inequality and interpolation inequality) } \\
& \leq C_{16}\|\nabla u\|_{L^{\infty, 2}}^{1 / 2} \text {. }
\end{aligned}
$$

where $C_{16}$ depends on $\alpha, \gamma$ and $\left\|u_{0}\right\|_{L^{2}}$.

For $\alpha=2$ and $\gamma=6$, from (36), we have

$$
\begin{aligned}
I_{5}^{1} & \leq \frac{1}{20}\|\Delta u\|_{L^{2,2}}^{2}+C_{17}\left\|\nabla u_{3}\right\|_{L^{\alpha, \gamma}}^{2}\|u\|_{L^{\infty, 3}}^{2} \\
& \leq \frac{1}{20}\|\Delta u\|_{L^{2,2}}^{2}+C_{17}\left\|\nabla u_{3}\right\|_{L^{\alpha, \gamma}}^{2}\|u\|_{L^{\infty, 2}}\|u\|_{L^{\infty, 6}} \\
& \leq \frac{1}{20}\|\Delta u\|_{L^{2,2}}^{2}+C_{18}\left\|\nabla u_{3}\right\|_{L^{\alpha, \gamma}}^{2}\|\nabla u\|_{L^{\infty, 2}}
\end{aligned}
$$

where $C_{18}$ only depends on $\left\|u_{0}\right\|_{L^{2}}$.

So for $2 \leq \alpha<4$, we have

$$
I_{5}^{1} \leq \frac{1}{20}\|\Delta u\|_{L^{2,2}}^{2}+C_{19}\left\|\nabla u_{3}\right\|_{L^{\alpha, \gamma}}^{2}\|\nabla u\|_{L^{\infty, 2}}
$$

where $C_{19}$ depends only on $\alpha, \gamma$ and $\left\|u_{0}\right\|_{L^{2}}$.

Then we turn our attention to $4 / 3<\alpha<2$. Actually it is more difficult than the 
previous case.

$$
\begin{aligned}
I_{5}^{1} & =\int_{0}^{t} \int_{\mathbb{R}^{3}}\left|\left(\partial_{2} u_{3}\right) u_{2} \Delta u_{3}\right| d x d \tau \\
& \leq \int_{0}^{t}\|\Delta u\|_{L^{2}}\left\|\nabla u_{3}\right\|_{L^{b}}\left\|u_{2}\right\|_{L^{a}} d \tau \\
& \leq \int_{0}^{t}\|\Delta u\|_{L^{2}}\left\|\nabla u_{3}\right\|_{L^{\gamma}}^{\delta}\|\nabla u\|_{L^{p}}^{1-\delta}\|u\|_{L^{a}} d \tau \\
& \leq C_{20} \int_{0}^{t}\|\Delta u\|_{L^{2}}\left\|\nabla u_{3}\right\|_{L^{\gamma}}^{\delta}\|\nabla u\|_{L^{2}}^{(1-\delta) \theta}\|\Delta u\|_{L^{2}}^{(1-\theta)(1-\delta)}\|u\|_{L^{a}} d \tau \\
& \leq \frac{1}{20}\|\Delta u\|_{L^{2,2}}^{2}+C_{21} \int_{0}^{t}\left\|\nabla u_{3}\right\|_{L^{\gamma}}^{2 \delta /(\theta+\delta-\theta \delta)}\|\nabla u\|_{L^{2}}^{2 \theta(1-\delta) /(\theta+\delta-\theta \delta)}\|u\|_{L^{a}}^{2 /(\theta+\delta-\theta \delta)} d \tau \\
& \leq \frac{1}{20}\|\Delta u\|_{L^{2,2}}^{2}+C_{21}\left\|\nabla u_{3}\right\|_{L^{\alpha, \gamma}}^{2 \delta /(\theta+\delta-\theta \delta)}\|\nabla u\|_{L^{\infty, 2}}^{2 \theta(1-\delta) /(\theta+\delta-\theta \delta)}\|u\|_{L^{q, a}}^{2 /(\theta+\delta-\theta \delta)}
\end{aligned}
$$

where the constants satisfy the following system

$$
\left\{\begin{array}{l}
\frac{1}{a}+\frac{1}{b}=\frac{1}{2} \\
\frac{1}{b}=\frac{\delta}{\gamma}+\frac{1-\delta}{p} \\
\frac{1}{p}=(1-\theta)\left(\frac{1}{2}-\frac{1}{3}\right)+\frac{1}{2} \theta \\
\frac{2 \delta}{\theta+\delta-\theta \delta} \times \frac{1}{\alpha}+\frac{2}{\theta+\delta-\theta \delta} \times \frac{1}{q}=1
\end{array}\right.
$$

It is obvious that the system is under determined since 4 equations and 6 unknowns. How can we find other equations?

Before solving (38), one can calculate directly from (38) that

$$
\frac{2}{q}+\frac{3}{a}=1
$$

therefore, as the above estimate, it is not difficult to obtain

$$
\|u\|_{L^{q, a}} \leq C\left(q, a, \theta, \delta,\left\|u_{0}\right\|_{L^{2}}\right)\|\nabla u\|_{L^{\infty, 2}}^{1 / 2},
$$

actually one can choose $q=\infty$, and a natural requirement of $\theta, \delta$ is as follows

$$
\frac{1+2 \theta(1-\delta)}{\theta+\delta-\theta \delta}=2
$$

Now, we can solve (38) with

$$
\left\{\begin{array}{l}
\delta=\frac{1}{2} \\
\theta=\frac{2}{\alpha}-1 \\
p=\frac{6 \alpha}{4-\alpha} \\
a=3 \\
b=6 \\
q=\infty
\end{array}\right.
$$

Therefore

$$
\begin{aligned}
I_{5}^{1} & \leq \frac{1}{20}\|\Delta u\|_{L^{2,2}}^{2}+C_{21}\left\|\nabla u_{3}\right\|_{L^{\alpha, \gamma}}^{\alpha}\|\nabla u\|_{L^{\infty, 2}}^{2-\alpha}\|u\|_{L^{\infty, 3}}^{2 \alpha} \\
& \leq \frac{1}{20}\|\Delta u\|_{L^{2,2}}^{2}+C_{21}\left\|\nabla u_{3}\right\|_{L^{\alpha, \gamma}}^{\alpha}\|\nabla u\|_{L^{\infty, 2}}^{2-\alpha}\|u\|_{L^{\infty, 2}}^{\alpha}\|u\|_{L^{\infty, 6}}^{\alpha} \\
& \leq \frac{1}{20}\|\Delta u\|_{L^{2,2}}^{2}+C_{22}\left\|\nabla u_{3}\right\|_{L^{\alpha, \gamma}}^{\alpha}\|\nabla u\|_{L^{\infty, 2}}^{2}
\end{aligned}
$$


where $C_{21}$ depends on $\alpha, \gamma$ and $\left\|u_{0}\right\|_{L^{2}}$

$$
\begin{aligned}
I_{5}^{2} & =\left|\int_{0}^{t} \int_{\mathbb{R}^{3}}\left(\partial_{3} u_{3}\right) u_{2} \Delta u_{3} d x d \tau\right|=\left|\int_{0}^{t} \int_{\mathbb{R}^{3}} \frac{1}{2} u_{2}^{2} \Delta\left(\partial_{3} u_{3}\right) d x d \tau\right| \\
& \leq\left|\int_{0}^{t} \int_{\mathbb{R}^{3}}\left(\partial_{3} u_{3}\right) u_{2} \Delta u_{2} d x d \tau\right|+\left.\left|\int_{0}^{t} \int_{\mathbb{R}^{3}}\left(\partial_{3} u_{3}\right)\right| \nabla u_{2}\right|^{2} d x d \tau \mid \equiv I_{5}^{2,1}+I_{5}^{2,2}
\end{aligned}
$$

$I_{5}^{2,1}$ can be treated similarly as $I_{5}^{1}$. For $2 \leq \alpha<4$,

$$
I_{5}^{2,1} \leq \frac{1}{20}\|\Delta u\|_{L^{2,2}}^{2}+C_{19}\left\|\nabla u_{3}\right\|_{L^{\alpha, \gamma}}^{2}\|\nabla u\|_{L^{\infty, 2},},
$$

while for $4 / 3<\alpha<2$,

$$
\begin{aligned}
I_{5}^{2,1} & \leq \frac{1}{20}\|\Delta u\|_{L^{2,2}}^{2}+C_{22}\left\|\nabla u_{3}\right\|_{L^{\alpha, \gamma}}^{\alpha}\|\nabla u\|_{L^{\infty, 2},}^{2} . \\
I_{5}^{2,2} & \leq\left.\int_{0}^{t} \int_{\mathbb{R}^{3}}\left|\left(\partial_{3} u_{3}\right)\right| \nabla u_{2}\right|^{2} \mid d x d \tau \\
& \leq\left\|\nabla u_{3}\right\|_{L^{\alpha, \gamma} \gamma}\|\nabla u\|_{L^{2 \alpha /(\alpha-1), 2 \gamma /(\gamma-1)}}^{2} \\
& \leq\left\|\nabla u_{3}\right\|_{L^{\alpha, \gamma}}\|\nabla u\|_{L^{2,2}}^{1 / 2}\|\nabla u\|_{L^{6 \alpha /(3 \alpha-4), 6 \gamma /(3 \gamma-4)}}^{3 / 2} \\
& \leq C_{23}\left\|\nabla u_{3}\right\|_{L^{\alpha, \gamma}}\|\nabla u\|_{L^{\infty, 2}}^{2 / \alpha}\|\Delta u\|_{L^{2,2}}^{3 / \gamma} .
\end{aligned}
$$

where $C_{23}$ depends on $\gamma$ and $\left\|u_{0}\right\|_{L^{2}}$.

Similarly, for $2 \leq \alpha<4$,

$$
\begin{aligned}
& I_{6} \leq \frac{1}{10}\|\Delta u\|_{L^{2,2}}^{2}+2 C_{19}\left\|\nabla u_{3}\right\|_{L^{\alpha, \gamma}}^{2}\|\nabla u\|_{L^{\infty, 2}} \\
& C_{23}\left\|\nabla u_{3}\right\|_{L^{\alpha, \gamma}}\|\nabla u\|_{L^{\infty, 2}}^{2 / \alpha}\|\Delta u\|_{L^{2,2}}^{3 / \gamma},
\end{aligned}
$$

while for $4 / 3<\alpha<2$,

$$
\begin{aligned}
I_{6} \leq & \frac{1}{10}\|\Delta u\|_{L^{2,2}}^{2}+2 C_{22}\left\|\nabla u_{3}\right\|_{L^{\alpha, \gamma}}^{\alpha}\|\nabla u\|_{L^{\infty, 2}}^{2} \\
& +C_{23}\left\|\nabla u_{3}\right\|_{L^{\alpha, \gamma}}\|\nabla u\|_{L^{\infty, 2}}^{2 / \alpha}\|\Delta u\|_{L^{2,2}}^{3 / \gamma} .
\end{aligned}
$$

For $2 \leq \alpha<4$, putting (31), (33), (34), (35), (37), (40), (42) and (43) into (30), one obtain

$$
\begin{aligned}
& \frac{1}{2}\|\nabla u\|_{L^{2}}^{2}+\|\Delta u\|_{L^{2,2}}^{2} \\
& \leq \frac{2}{5}\|\Delta u\|_{L^{2,2}}^{2}+2 C_{8}\left\|\nabla u_{3}\right\|_{L^{\alpha, \gamma}}^{\alpha}\|\nabla u\|_{L^{\infty, 2}}^{2} \\
& +2 C_{12}\|\nabla u\|_{L^{\infty, 2}}^{2-3 / \gamma}\|\Delta u\|_{L^{2,2}}^{3 / \gamma}\left\|\nabla u_{3}\right\|_{L^{\alpha, \gamma}}^{2} \\
& +4 C_{19}\left\|\nabla u_{3}\right\|_{L^{\alpha, \gamma}}^{2}\|\nabla u\|_{L^{\infty, 2}}+\frac{1}{2}\left\|\nabla u_{0}\right\|_{L^{2}}^{2} \\
& +2 C_{23}\left\|\nabla u_{3}\right\|_{L^{\alpha, \gamma}}\|\nabla u\|_{L^{\infty, 2}}^{2 / \alpha}\|\Delta u\|_{L^{2,2}}^{3 / \gamma}+2 C_{13}\|\nabla u\|_{L^{\infty, 2}} \\
& \leq \frac{1}{2}\|\Delta u\|_{L^{2,2}}^{2}+2\left(C_{8}\left\|\nabla u_{3}\right\|_{L^{\alpha, \gamma}}^{\alpha}+C_{24}\left\|\nabla u_{3}\right\|_{L^{\alpha, \gamma}}^{4 /(2-3 / \gamma)}\right)\|\nabla u\|_{L^{\infty, 2}}^{2} \\
& +4 C_{19}\left\|\nabla u_{3}\right\|_{L^{\alpha, \gamma}}^{2}\|\nabla u\|_{L^{\infty, 2}} \\
& +2 C_{25}\left\|\nabla u_{3}\right\|_{L^{\alpha, \gamma}}^{\frac{2 \gamma}{2 \gamma-3}}\|\nabla u\|_{L^{\infty, 2}}^{2-\frac{\gamma}{2 \gamma-3}}+2 C_{13}\|\nabla u\|_{L^{\infty, 2}}+\frac{1}{2}\left\|\nabla u_{0}\right\|_{L^{2}}^{2} .
\end{aligned}
$$


For $2 \leq \alpha<4$, putting (31), (33), (34), (35), (39), (41), (42) and (44) into (30), one obtain

$$
\begin{aligned}
& \frac{1}{2}\|\nabla u\|_{L^{2}}^{2}+\|\Delta u\|_{L^{2,2}}^{2} \\
& \leq \frac{2}{5}\|\Delta u\|_{L^{2,2}}^{2}+2 C_{8}\left\|\nabla u_{3}\right\|_{L^{\alpha, \gamma}}^{\alpha}\|\nabla u\|_{L^{\infty, 2}}^{2} \\
& +2 C_{12}\|\nabla u\|_{L^{\infty, 2}}^{2-3 / \gamma}\|\Delta u\|_{L^{2,2}}^{3 / \gamma}\left\|\nabla u_{3}\right\|_{L^{\alpha, \gamma}}^{2} \\
& 4 C_{22}\left\|\nabla u_{3}\right\|_{L^{\alpha, \gamma}}^{\alpha}\|\nabla u\|_{L^{\infty, 2}}^{2} \\
& +2 C_{23}\left\|\nabla u_{3}\right\|_{L^{\alpha, \gamma}}\|\nabla u\|_{L^{\infty, 2}}^{2 / \alpha}\|\Delta u\|_{L^{2,2}}^{3 / \gamma}+2 C_{13}\|\nabla u\|_{L^{\infty, 2}} \\
& \leq \frac{1}{2}\|\Delta u\|_{L^{2,2}}^{2}+2\left(\left(C_{8}+2 C_{22}\right)\left\|\nabla u_{3}\right\|_{L^{\alpha, \gamma}}^{\alpha}+C_{26}\left\|\nabla u_{3}\right\|_{L^{\alpha, \gamma}}^{4 /(2-3 / \gamma)}\right)\|\nabla u\|_{L^{\infty, 2}}^{2} \\
& +4 C_{19}\left\|\nabla u_{3}\right\|_{L^{\alpha, \gamma}}^{2}\|\nabla u\|_{L^{\infty, 2}} \\
& +2 C_{27}\left\|\nabla u_{3}\right\|_{L^{\alpha, \gamma}}^{\frac{2 \gamma}{2 \gamma-3}}\|\nabla u\|_{L^{\infty, 2}}^{2-\frac{\gamma}{2 \gamma-3}}+2 C_{13}\|\nabla u\|_{L^{\infty, 2}}+\frac{1}{2}\left\|\nabla u_{0}\right\|_{L^{2}}^{2} .
\end{aligned}
$$

Just as the proof for the case $\gamma=3$, using the integrability of $\left\|u_{3}\right\|_{L^{\gamma}}$ with respect to time variable, we can choose a sufficiently small $t_{0}, 0<t_{0} \leq T$, such that

$$
C_{8} \int_{0}^{t_{0}}\left\|\nabla u_{3}(., \tau)\right\|_{L^{\gamma}}^{\alpha} d \tau+C_{24}\left(\int_{0}^{t_{0}}\left\|\nabla u_{3}(. \tau)\right\|_{L^{\gamma}}^{\alpha} d \tau\right)^{\frac{4}{\alpha(2-3 / \gamma)}} \leq \frac{1}{4}
$$

Due to (45) and (47),

$$
\begin{aligned}
& \frac{1}{4}\|\nabla u\|_{L^{\infty, 2}}^{2}+\frac{1}{2}\|\Delta u\|_{L^{2,2}}^{2} \\
& \leq 4 C_{19}\left\|\nabla u_{3}\right\|_{L^{\alpha, \gamma}}^{2}\|\nabla u\|_{L^{\infty, 2}}+\frac{1}{2}\left\|\nabla u_{0}\right\|_{L^{2}}^{2} \\
& \quad+2 C_{25}\left\|\nabla u_{3}\right\|_{L^{\alpha, \gamma}}^{\frac{2 \gamma}{2 \gamma-3}}\|\nabla u\|_{L^{\infty, 2}}^{2-\frac{\gamma}{2-3}}+2 C_{13}\|\nabla u\|_{L^{\infty, 2}}
\end{aligned}
$$

Since the power of $\|\nabla u\|_{L^{\infty, 2}}$ in the right side of (48) is strictly less than 2 , so we immediately have the estimate

$$
\|\nabla u\|_{L^{\infty, 2}}^{2}+\|\Delta u\|_{L^{2,2}}^{2} \leq C_{28},
$$

where $C_{28}$ depends on $\alpha, \gamma,\left\|u_{0}\right\|_{L^{2}}$ and $\left\|\nabla u_{0}\right\|_{L^{2}}$. Then the remaining argument is same as that in the proof of Lemma 4.

Similar argument can be done for $4 / 3<\alpha<2$. This finishes the proof of Lemma 6. $\square$

After we have the a priori estimates Lemma 6, the proof of Theorem 1 is completely similar to the case when $\gamma=3$.

Therefore, we finish the proof of Theorem 1.

Acknowledgment. The author would like to express sincere gratitude to his supervisor Professor Zhouping Xin for enthusiastic guidance and constant encouragement. And thanks also to Professor Shing-Tung Yau and Professor Zhouping Xin for providing an excellent study and research environment in The Institute of Mathematical Sciences. 


\section{REFERENCES}

[1] H. Beirão Da Veiga, A new regularity class for the Navier-Stokes equations in $\mathbb{R}^{n}$, Chinese Ann. Math., 16 (1995), pp. 407-412.

[2] L. Caffarelli, R. Kohn, L. Nirenberg, Partial regularity of suitable weak solutions of the Navier-Stokes equations, Comm. Pure Appl. Math., 35 (1982), pp. 771-831.

[3] D. Chat, H-J. ChoE, On the regularity criterion for the solutions of the Navier-Stokes equations, Electron. J. Differential Equations 1999, (05) (1999), pp. 1-7.

[4] D. Chat, J. LeE, Regularity criterion in terms of pressure for the Navier-Stokes equations, Nonlinear Analysis, 46 (2001), pp. 727-735.

[5] Y. GigA, Solutions for semilinear parabolic equations in $L^{p}$ and regularity of weak solutions of the Navier-Stokes system, J. Differential Equations, 62 (1986), pp. 186-212.

[6] C. HE, Regularity for solutions to the Navier-Stokes equations with one velocity component regular, Electron. J. Differential Equations, 29 (2002), pp. 1-13.

[7] E. Hopf, Über die Anfangwertaufgaben für die hydromischen Grundgleichungen, Math. Nach., 4 (1951), pp. 213-321.

[8] H. Kozono, H. Sohr, Regularity criterion on weak solutions to the Navier-Stokes equations, Adv. Differential Equations, 2 (1997), pp. 535-554.

[9] H. Kozono, Y. TAniuchi, Bilinear estimates in BMO and the Navier-Stokes equations, Math. Z., 235 (2000), pp. 173-194.

[10] J. LERAY, Étude de divers équations intégrales nonlinearies et de quelques problemes que posent lhydrodinamique, J. Math. Pures. Appl., 12 (1933), pp. 1-82.

[11] K. MAsudA, Weak solutions of the Navier-Stokes equations, Tohoku Math. J., 36 (1984), pp. 623-646.

[12] V. Scheffer, Partial regularity of solutions to the Navier-Stokes equations, Pacific J. Math., 66 (1976), pp. 535-552.

[13] J. SERRIN, On the interior regularity of weak solutions of the Navier-Stokes equations, Arch. Rational Mech. Anal., 9 (1962), pp. 187-195.

[14] H. SoHR, A regularity class for the Navier-Stokes equations in Lorentz spaces, J. Evolution Equations, 1 (2001), pp. 441-467.

[15] H. SoHr, A generalization of Serrin's regularity criterion for the Navier-Stokes equations, Quaderni Di Math., (2002), to appear.

[16] M. Struwe, On partial regularity results for the Navier-Stokes equations, Comm. Pure Appl. Math., 41 (1988), pp. 437-458.

[17] G. Tian, Z. XIN, Gradient Estimation on Navier-Stokes equations, Comm. Anal. Geo., 7 (1999), pp. 221-257.

[18] W. VON WAHL, Regularity of weak solutions of the Navier-Stokes equations, Proceedings of the 1983 Summer Institute on Nonlinear Functional Analysis and Applications, Proc. Symposia in Pure Mathematics 45, Providence Rhode Island: Amer. Math. Soc. (1986), pp. 497-503.

[19] W. VON WAHL, The equations of Navier-Stokes and abstract parabolic equations, Aspects of Mathematics, No. E8, Vieweg, Braunschweig, 1985.

[20] Y. Zhou, Regularity criteria in terms of pressure for the Navier-Stokes Equations in $\mathbb{R}^{3}$, Preprint, (2002).

[21] Y. Zноu, Some new regularity criteria of weak solutions to the Navier-Stokes equations, Preprint, (2002). 